\title{
Cooling of the Earth: A parameterized convection study of whole versus layered models
}

\author{
Allen K. McNamara and Peter E. van Keken \\ Department of Geological Sciences, 2534 C.C. Little Building, 425 East University, University of Michigan, Ann \\ Arbor, Michigan 48109-1063,USA (mcnamar@umich.edu; keken@umich.edu)
}

[1] Compositionally layered mantle models have often been invoked in order to explain the geochemistry observed at the Earth's surface, specifically the discrepancy between ocean island basalt and mid-ocean ridge basalt compositions. One disadvantage of layered models is the reduction in cooling efficiency compared to whole-mantle convection as a direct result of the insulating nature of the thermal boundary layer that develops between the two convecting layers. This may pose a significant problem for layered models in which the bottom layer is enriched in heat-producing radioactive elements with respect to the top layer. One may expect that the bottom layer would become superheated over the lifetime of the Earth. We perform this study in order to test whether layered models are thermally feasible. We are interested in discovering whether it was possible, within Earth-like constraints, to produce a bottom layer temperature that remains below the solidus without simultaneously producing a top layer that is too cool. We use parameterized convection to explore a wide parameter range of input values for several layering configurations. We study both a whole mantle convection model and the recently proposed layered convection model, which places the boundary between the layers at 1600-km depth [Kellogg et al., 1999]. We use the present-day heat flow and mantle viscosity as primary constraints and use the resulting average temperature as a test for the feasibility of the models. Our results reveal that for whole mantle convection, a wide parameter range produces results that satisfy our constraints. This is in contrast to the layered convection model in which we find that the parameter range that satisfies constraints is significantly reduced or perhaps, nonexistent.

Components: 7408 words, 5 figures, 3 tables.

Keywords: mantle; convection; parameterized; thermal evolution; layered convection; temperature.

Index Terms: 8125 Tectonophysics: Evolution of the Earth (0325); 1212 Geodesy and Gravity: Earth's interior: composition and state $(7207,7208,8105,8124) ; 8130$ Tectonophysics: Heat generation and transport.

Received 6 February 2000; Revised 18 August 2000; Accepted 29 September 2000; Published 13 November 2000.

McNamara, A. K., and P. E. van Keken (2000), Cooling of the Earth: A parameterized convection study of whole versus layered models, Geochem. Geophys. Geosyst., 1, 1027, doi:10.1029/2000GC000045. 


\section{Introduction}

[2] Geochemical differences between basalts formed at mid-ocean ridges (MORBs) and ocean islands (OIBs) have intimated at the presence of at least two distinct geochemical reservoirs in the Earth's mantle [Hofmann, 1997]. MORBs are relatively homogeneous on a global scale and are depleted in incompatible elements in contrast to OIBs, which are observed to be geochemically heterogeneous yet are less depleted or enriched in incompatible elements with respect to MORBs. Because midocean ridges are thought to adequately sample the upper mantle spatially through time, it has been proposed that the upper mantle consists of a depleted reservoir, and source material for OIBs originates from a deeper reservoir and is brought to the surface via mantle plumes [e.g., Morgan, 1971]. Further weight is given to such considerations by calculations of the thermal budget of the Earth. The present-day heat flow of the Earth may be explained by a combination of secular cooling and radiogenic heating. The required amount of radiogenic heating is approximately that of the bulk silicate earth (BSE) [Van Schmus, 1995] with a much higher concentration of radioactive elements than that observed in the MORB source [Van Schmus, 1995; Hofmann, 1997]. It is therefore attractive to imagine a reservoir of concentrated radioactive material in the deep Earth that is not sampled by mid-oceanic ridges.

[3] This line of arguments has led to the "classical" layered geochemical model in which the ringwoodite to perovskite + magnesiowüstite phase transition at $670-\mathrm{km}$ depth was inferred to be the boundary between an upper depleted layer and an enriched lower layer. A number of geophysical observations, however, have led to a major rethinking of this model. Seismic tomography models provide the most convincing evidence in that they reveal subducted slabs are able to penetrate the lower mantle [Grand et al., 1997; van der Hilst et al., 1997; Bijwaard et al., 1998] and that mantle plumes may originate at the core-mantle boundary [Bijwaard and Spakman, 1999] or at least below 670-km depth [Shen et al., 1998]. In addition, there are modeldependent arguments that point to difficulties associated with a major thermal boundary layer at $670-\mathrm{km}$ depth. For example, numerical calculations have revealed that the presence of a thermal boundary layer at $670-\mathrm{km}$ depth would produce a significant amount of topography comparable to that due to the cooling lithosphere as well as large positive geoid and gravity anomalies. These are not observed [Davies and Richards, 1992]. Furthermore, parameterized convection results show that the insulating effect of such a boundary layer tends to raise the lower-layer temperature significantly above its melting temperature unless a strong depletion in radioactive elements with respect to the upper mantle is imposed [Spohn and Schubert, 1982]. This is contrary to the main assumption of radioactive element enrichment in the lower layer.

[4] In order to reconcile a layered model with seismic observations, Kellogg et al. [1999] propose a layered model that places the boundary between the layers deeper in the mantle at $1600-\mathrm{km}$ depth. Their model may explain the interpretation of some tomographic results [van der Hilst et al., 1997] and can be consistent with the inferred nature of seismic heterogeneity observed below 1600-km depth [van der Hilst and Karason, 1999]. Their dynamical calculations indicate that an intrinsic density of $5 \%$ allows the lower layer to remain stable and produces a net density contrast allowable within mineral physics constraints.

[5] It is expected that such a model would necessitate a substantial enrichment of radioactive elements in the lower layer to account for the present-day mantle heat loss of $40 \mathrm{TW}$ (inferred by assuming that $10 \%$ of the total heat 
loss, 44.2 TW [Pollack et al., 1993], originates in the continental crust). As a consequence, one could expect that a significant boundary layer will develop. In this paper, we will investigate the feasibility of such layered models in which heat-producing elements are concentrated in the lower layer. Our approach will be to use parameterized convection models that satisfy the present-day heat flow and fall within the range of estimates for mantle viscosity and average mantle temperature. In effect, we perform a Monte Carlo inversion of the parameters that govern the convection models to depict an admissible parameter range. In particular, we determine the necessary heat production and rheological formulation for a range of governing dynamical parameters such as the power law exponent, $\beta$, and the critical Rayleigh number, $R a_{c}$.

[6] We start with a whole-mantle convection model and compare results to the proposed layered model in which the boundary is located at $1600-\mathrm{km}$ depth.

\section{Parameterized Modeling Approach}

[7] Parameterized convection is not as powerful as full dynamical calculations, but it does allow a computationally inexpensive method to explore the parameter range appropriate for the Earth. This is especially beneficial when dealing with high Rayleigh numbers on timescales of the age of the Earth.

[8] The ease at which parameterized convection allows an inspection of large parameter spaces has prompted its use to study the Earth's thermal history in both whole-mantle models [e.g., Turcotte, 1980; Schubert et al., 1980; Jackson and Pollack, 1984] as well as models that were layered at $670-\mathrm{km}$ depth [e.g., McKenzie and Richter, 1981; Spohn and Schubert, 1982; Honda, 1995]. One possible criticism in using this approach lies in the approximation of negligible topography between the layers. Examination of the results of Kellogg et al. [1999] reveals an extensive amount of topography on the boundary between the two layers; however, it is likely that this degree of topography is largely due to the unusually large thickness of their generated slab (500-1000 km when taking the reflective boundary condition into account). A more Earth-like vigor of convection has been shown numerically to significantly reduce the amount of topography due to decreasing thicknesses of upwellings and downwellings associated with increased Rayleigh number.

[9] The fundamental premise underlying parameterized convection is that the boundary layer thickness, $\delta$, may be represented as

$$
\delta=D\left(\frac{R a_{c}}{R a}\right)^{\beta}
$$

where $D$ is the thickness of the convecting layer, $R a_{c}$ is the critical Rayleigh number, $R a$ is the Rayleigh number, and the exponent $\beta$ is a constant. Some formulations of (1) include a constant of proportionality; when necessary, we choose to incorporate this constant into the $R a_{c}$ term. The Rayleigh number is expressed as

$$
R a=\frac{\alpha \rho g D^{3} \triangle T}{\kappa \eta(T)}
$$

where $\alpha$ is the thermal expansivity, $\rho$ is the density, $g$ is the gravitational constant, $\Delta T$ is the temperature drop across the convecting layer, $\kappa$ is the thermal diffusivity, and $\eta(T)$ is the viscosity at the average temperature.

[10] Equation (1) may be represented in terms of the heat flow $q$ :

$$
q=\frac{K \triangle T_{\delta}}{D}\left(\frac{R a}{R a_{c}}\right)^{\beta}
$$

where $\Delta T_{\delta}$ is the temperature drop across the thermal boundary layer and $k$ is the thermal conductivity. 
[11] The appropriate values of $\beta$ and $R a_{c}$ have been a matter of debate. Earlier, thermal evolution models applying a temperature-dependent viscosity have commonly used $\beta$ values ranging from 0.228 to 0.33 [e.g., Schubert et al., 1980; Spohn and Schubert, 1982; Jackson and Pollack, 1984; Williams and Pan, 1992] based on laboratory experiments and boundary layer theory. In contrast, Christensen [1984, 1985a, 1985b] determined a weak coupling between the Nusselt and Rayleigh numbers, specifically for temperature-dependent rheology, resulting in a value of $\beta<0.10$. Gurnis [1989] countered that the low $\beta$ values determined by Christensen were invalid because the strong temperature dependence leads to a style of rigid lid convection not present on the Earth. By incorporating weak zones to initiate subduction, Gurnis' dynamical models resulted in $\beta$ values of 0.3 . Later, laboratory experiments performed by Giannandrea and Christensen [1993] resulted in a determination of $\beta=0.20$. Comparison to dynamical results has led Honda and Iwase [1996] to infer $0.30<\beta<0.40$. More recently, Conrad and Hager [1999] consider the importance of resistance to slab bending at subduction zones and show that $\beta$ may be a function of plate size and lithospheric strength, ranging from 0 to $1 / 3$.

[12] In light of the uncertainty regarding the value of $\beta$ we examine a range of values for $\beta$ and $R a_{c}$. As we will show, we can use our approach to limit the range of acceptable $\beta$ values.

\section{Whole-Mantle Convection}

\subsection{Model Setup}

[13] In the whole-mantle convection model, heat flow is determined by the temperature gradient across the upper thermal boundary layer (equation (3)). The thermal evolution is calculated by applying the conservation of energy. Essentially, the change in thermal energy is equal to the net energy entering the system plus energy produced by the decay of radioactive elements,

$$
V \operatorname{rr} c_{p} \frac{d T}{d t}=q_{\text {in }} A_{\text {bottom }}-q_{\text {out }} A_{\text {top }}+Q_{\text {produced }} V
$$

where $V$ is the volume, $\rho$ is the density, $c_{p}$ is the specific heat, $T$ is the average temperature, $Q$ is the heat production per unit volume, and $A_{\text {bottom }}$ and $A_{\text {top }}$ are the areas of the bottom and top boundaries, respectively.

[14] The heat production is represented as the sum of heat production calculated for each of the radioactive species: ${ }^{235} \mathrm{U},{ }^{238} \mathrm{U},{ }^{232} \mathrm{Th},{ }^{40} \mathrm{~K}$. For each layer the relative ratio of each of the species is held constant according to Turcotte and Schubert [1982]. The only variable parameter is the concentration of uranium. As a result, we can write

$$
\begin{gathered}
Q_{\text {total }}=\Sigma Q_{i} \\
Q_{i}=H_{i}^{\prime} R_{i}[U] \exp \left(\lambda_{i}\left(t_{o}-t\right)\right)
\end{gathered}
$$

where $Q_{i}$ is the heat production of each species, $H_{i}^{\prime}$ is the heat production per mass of radioactive material, $R_{i}$ is the ratio of the species' concentration to that of uranium, [U] is the uranium concentration, $\lambda_{i}$ is the decay constant, and $t_{o}$ is the age of the earth.

[15] Values used were taken from Turcotte and Schubert [1982] and are listed in Table 1, along with other values used in the calculations.

[16] We use a viscosity formulation based on thermally activated creep that can be written as

$$
\eta=\eta_{o} \exp \left(\frac{E}{R T}\right)
$$

where $\eta_{o}$ is a prefactor that may also be considered a minimum viscosity, $E$ is the activation energy, $R$ is the gas constant, and $T$ is the average layer temperature.

[17] As we will discuss later, we prefer to maintain an insulated bottom boundary in the layered models, so to remain consistent, we choose to use an insulated bottom for the whole-mantle 
Table 1. List of Parameters and Initial Conditions Used in the Parameterized Convection Calculations

\begin{tabular}{|c|c|c|c|}
\hline Parameter & Description & Value & Units \\
\hline$\alpha$ & thermal expansivity & $3 \times 10^{-5}$ & $\mathrm{~K}^{-1}$ \\
\hline$\rho$ & reference density & 4500 & $\mathrm{~kg} / \mathrm{m}^{3}$ \\
\hline$\Delta \rho$ & intrinsic density increase in bottom layer & $5 \%$ & \\
\hline $\mathrm{c}$ & specific heat & 1250 & $\mathrm{~J} /(\mathrm{kg} \mathrm{K})$ \\
\hline$k$ & thermal conductivity & 5.6 & $\mathrm{~W} /(\mathrm{m} \mathrm{K})$ \\
\hline$\kappa$ & thermal diffusivity & $\mathrm{k} /(\rho \mathrm{c})$ & $\mathrm{m}^{2} / \mathrm{s}$ \\
\hline$g$ & gravitational constant & 9.8 & $\mathrm{~m} / \mathrm{s}^{2}$ \\
\hline$t_{o}$ & age of the Earth & 4.6 & Byr \\
\hline$T_{s}$ & surface temperature & 273 & $\mathrm{~K}$ \\
\hline$T_{\mathrm{o}, \text { whole }}$ & initial temperature of whole layer & 3200 & $\mathrm{~K}$ \\
\hline$T_{\mathrm{o}, \text { top }}$ & initial temperature of top layer & 3000 & $\mathrm{~K}$ \\
\hline$T_{\mathrm{o}}$, bottom & initial temperature of bottom layer & 3500 & $\mathrm{~K}$ \\
\hline$D$ & thickness of mantle & 2880 & $\mathrm{~km}$ \\
\hline$\lambda_{238 U}$ & decay constant of ${ }^{238} U$ & 0.155 & $\mathrm{Byr}^{-1}$ \\
\hline$\lambda_{235} U$ & decay constant of ${ }^{235} \mathrm{U}$ & 0.985 & $\mathrm{Byr}^{-1}$ \\
\hline$\lambda_{232} \mathrm{Th}$ & decay constant of ${ }^{232} \mathrm{Th}$ & 0.0495 & $\mathrm{Byr}^{-1}$ \\
\hline$\lambda_{40} \mathrm{~K}$ & decay constant of ${ }^{40} \mathrm{~K}$ & 0.555 & $\mathrm{Byr}^{-1}$ \\
\hline $\mathrm{H}_{238}^{\prime} \mathrm{U}$ & heat production of ${ }^{238} \mathrm{U}$ & $9.37 \times 10^{-5}$ & $\mathrm{~W} / \mathrm{kg}$ \\
\hline $\mathrm{H}^{\prime}{ }_{235} \mathrm{U}$ & heat production of ${ }^{235} \mathrm{U}$ & $5.69 \times 10^{-4}$ & $\mathrm{~W} / \mathrm{kg}$ \\
\hline $\mathrm{H}_{232}^{\prime} T h$ & heat production of ${ }^{232} \mathrm{Th}$ & $2.69 \times 10^{-5}$ & $\mathrm{~W} / \mathrm{kg}$ \\
\hline $\mathrm{H}^{\prime}{ }_{40} \mathrm{In}$ & heat production of ${ }^{40} \mathrm{~K}$ & $2.79 \times 10^{-5}$ & $\mathrm{~W} / \mathrm{kg}$ \\
\hline $\mathrm{R}_{238}{ }^{43} \mathrm{~K}$ & ratio of ${ }^{238} U$ to $U$ (all layers) & 0.9927 & \\
\hline $\mathrm{R}_{235} \mathrm{U}$ & ratio of ${ }^{235} \mathrm{U}$ to $\mathrm{U}$ (all layers) & 0.0072 & \\
\hline $\mathrm{R}_{40} \mathrm{~K}$ & ratio of ${ }^{40} \mathrm{~K}$ to $\mathrm{U}$ (all layers) & 1.28 & \\
\hline $\mathrm{R}_{232 T h}{ }^{40} \mathrm{~L}$ & ratio of ${ }^{232} \mathrm{Th}$ to $\mathrm{U}$ (whole layer) & 4.01 & \\
\hline $\mathrm{R}_{232 T h}$ & ratio of ${ }^{232} \mathrm{Th}$ to $\mathrm{U}$ (top layer) & 2.5 & \\
\hline $\mathrm{R}_{232 T h}$ & ratio of ${ }^{232} \mathrm{Th}$ to $\mathrm{U}$ (bottom layer) & 4.0 & \\
\hline$[U]_{\text {top }}$ & uranium concentration in top layer & 7 & $\mathrm{ppb}$ \\
\hline
\end{tabular}

convection model as well. The application of an insulating bottom boundary condition implies that $q_{\text {in }}=0$ in the energy balance. We integrate the energy balance equation using a fourth-order Runge-Kutta integration scheme, starting with an initial temperature of $3200 \mathrm{~K}$.

[18] We systematically vary the input parameters, $\beta, R a_{c}$, and $E$. The parameter $\beta$ is varied from 0.10 to 0.33 , and two end-member choices of $R a_{c}$ were chosen as 1100 and 87.79. We choose $R a_{c}=1100$ to resemble the parameterization used by Jackson and Pollack [1984] and Spohn and Schubert [1982], and $R a_{c}=87.79$ translates to the Nusselt-Rayleigh relationship suggested by Turcotte and Schubert [1982] when $\beta=1 / 3$. We employ two endmember values of activation energy to mimic isoviscous $(E=0)$ and olivine-like rheology $(E$ $=525 \mathrm{~kJ} / \mathrm{mol}$; [Karato and $W u, 1993]$ ).
[19] For each set of above parameters we vary two additional input parameters: the viscosity prefactor, $\eta_{o}$, and the radioactive element concentration. These are varied until the output constraints of present-day viscosity, $\eta_{p d}$, and present-day heat loss are satisfied. In this process, thousands of runs are computed, but only those satisfying these output constraints are retained for further examination.

[20] We assume three different values of $\eta_{p d}$, which reflects the uncertainty in present-day mantle viscosity values [Peltier, 1989; Mitrovica, 1997]. We assume that $10 \%$ of the present-day global heat loss of $44.2 \mathrm{TW}$ is produced in the crust [Pollack and Chapman, 1977; Pollack et al., 1993], leaving a mantle heat loss of $40 \mathrm{TW}$. 
[21] We use the resultant temperature as the the primary test on the feasibility of the models. We reject parameter combinations that yield an average temperature exceeding $4000 \mathrm{~K}$ at any time during the model's thermal history. This estimate is higher than that used in other studies [Spohn and Schubert, 1982; Honda, 1995] because we wish to allow for the uncertainties in melting temperature of lower mantle material [e.g., Holland and Ahrens, 1997; Zerr et al., 1998; Wang, 1999]. We also reject models that have a present-day average temperature below $1500 \mathrm{~K}$, as this corresponds to the temperature at the base of the lithosphere.

\subsection{Results}

[22] Before discussing the parameterized convection results, it is interesting to note that under the constraints of present-day viscosity and heat flow, a simple analytical expression may be derived to determine the present-day average mantle temperature. Equations (2) and (3) can be rearranged as

$$
T=\left(\frac{q D}{K}\left(\frac{\kappa \eta(T) R a_{c}}{\alpha g \rho D^{3}}\right)^{\beta}\right)^{\frac{1}{1+\beta}}+T_{s}
$$

For this we have used $\Delta T_{\delta}=\Delta T=T-T_{s}$, where $T$ is the average mantle temperature and $T_{S}$ is the surface temperature. For present-day values, which are indicated by the subscript $p d$,

$$
T_{p d}=\left(\frac{q_{p d} D}{K}\left(\frac{\kappa \eta_{p d} R a_{c}}{\alpha g \rho D^{3}}\right)^{\beta}\right)^{\frac{1}{1+\beta}}+T_{s} .
$$

[23] Note that the present-day temperature is independent of initial temperature and activation energy and only weakly dependent on $\kappa, \eta_{p d}, R a_{c}, \alpha$, and $\rho$ for low values of $\beta$. Although heat production is not expressed within the equation, it is strongly linked to the present-day heat flow.

[24] The thermal evolution of two parameterized convection calculations along with heat flow and heat production are shown in Figure 1. The input parameters, $\beta$ and $R a_{c}$ and the output constraint $\eta_{p d}=10^{21} \mathrm{~Pa} \mathrm{~s}$ are identical for each run. The difference between the two calculations is the rheology $(E=0$ and $E=525 \mathrm{~kJ} / \mathrm{mol})$ and, as a consequence, the values of $\eta_{o}$ and [U] required to constrain the present-day output parameters of viscosity and heat flow. Note that although the final temperature is essentially identical for each calculation, the thermal evolution as well as the heat production are quite distinct. The strongly temperature-dependent rheology in the $E=525 \mathrm{~kJ} / \mathrm{mol}$ case causes a rapid initial cooling as illustrated by the precipitous decrease in temperature and high values of heat flow. After the initial cooling, the temperature decreases slowly. In contrast, the isoviscous case experiences a more prolonged period of slow cooling and overall lower heat flow. The lower convective vigor of the isoviscous case throughout most of its history results in a lower radioactive element concentration required to produce the present-day heat flow. This is due to the ability of the isoviscous system to retain much of its radiogenic heat over time in contrast to the negative-feedback nature of the temperature-dependent rheology that facilitates rapid removal of excess heat. This effect is illustrated by the difference in Urey ratio for both cases. The Urey ratio is the ratio of present-day heat production over present-day heat flow. The isoviscous case yields a low Urey ratio of 0.17 , indicating low heat production and a high degree of secular cooling, whereas the temperature-dependent case leads to a high Urey ratio of 0.88 , predicting a near balance between heat production and heat loss.

[25] This brings attention to the difficulty involved in using parameterized convection to study the radioactive element composition of the Earth. Our results show that present-day constraints may be satisfied with contrasting rheologies; however, the concentration of radioactive elements is highly dependent on activation energy. 

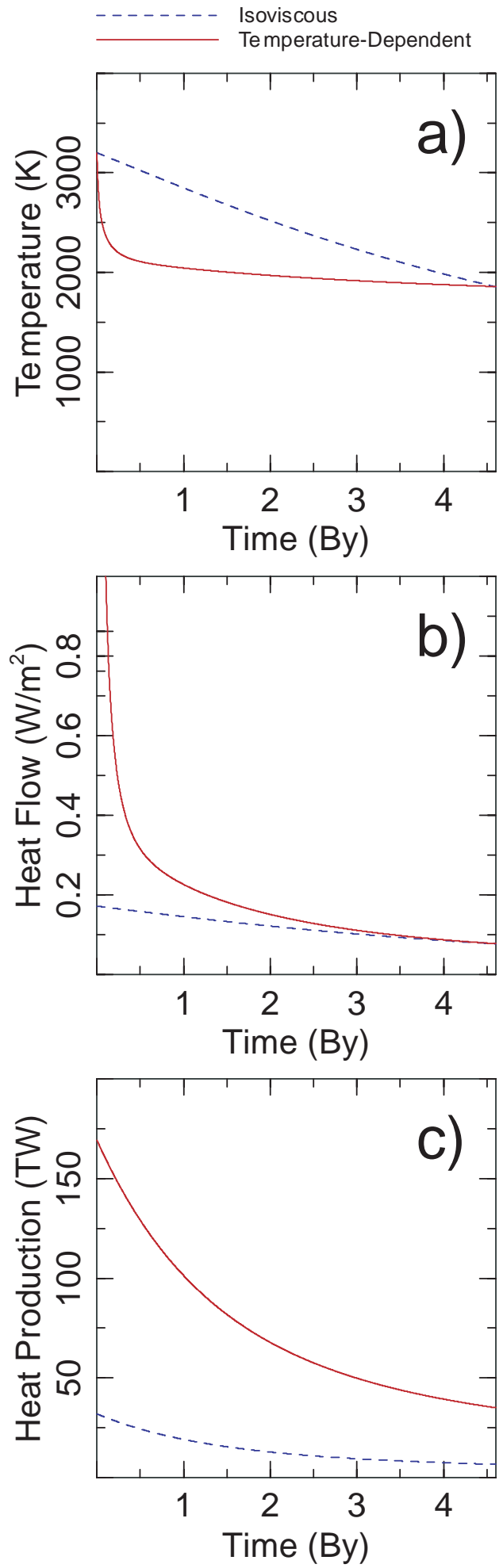

[26] Parameterized convection results for all whole-mantle runs are plotted in Figure 2 for both values of $R a_{c}$. The curves represent the present-day temperatures calculated from the analytical equation described above for the three constraints of present-day viscosity. Superimposed on these as solid symbols are present-day temperatures determined from parameterized convection calculations. These results are obtained by using the temperaturedependent rheology, but examination of Table 1 reveals similar temperatures for the isoviscous rheology, as expected. Horizontal lines at 1500 and $4000 \mathrm{~K}$ mark the boundaries of the temperature range that we find acceptable. Following our expectations, we observe that low values of $\beta$ result in excessively high present-day mantle temperatures owing to the reduction in cooling efficiency. Similarly, higher present-day viscosity constraints result in higher present-day mantle temperatures. Reducing the value of $R a_{c}$ leads to more efficient cooling and consequently to lower temperatures for a given value of $\beta$, but it is observed that a relatively large change in $R a_{c}$ from 1100 to 87.79 leads to only a minor shift in the final temperature toward lower values of $\beta$.

[27] Table 2 lists the parameters used in the cases that produce acceptable temperatures. The concentration of uranium required to satisfy the present-day heat loss is compared with that of the bulk silicate Earth (BSE), with a given uranium concentration of $21 \mathrm{ppm}$ by Van Schmus [1995].

Figure 1. (a) Temperature, (b) heat flow, and (c) heat production results from two whole-mantle parameterized convection calculations. Both use $R a_{c}=1100, \eta\left(\mathrm{T}_{p d}\right)=10^{21} \mathrm{~Pa} \cdot \mathrm{s}$, and $\beta=0.30$. One of the calculations utilizes an isoviscous rheology $(E=0 \mathrm{~kJ} / \mathrm{mol})$ and the other has a temperaturedependent one $(E=525 \mathrm{~kJ} / \mathrm{mol})$. 

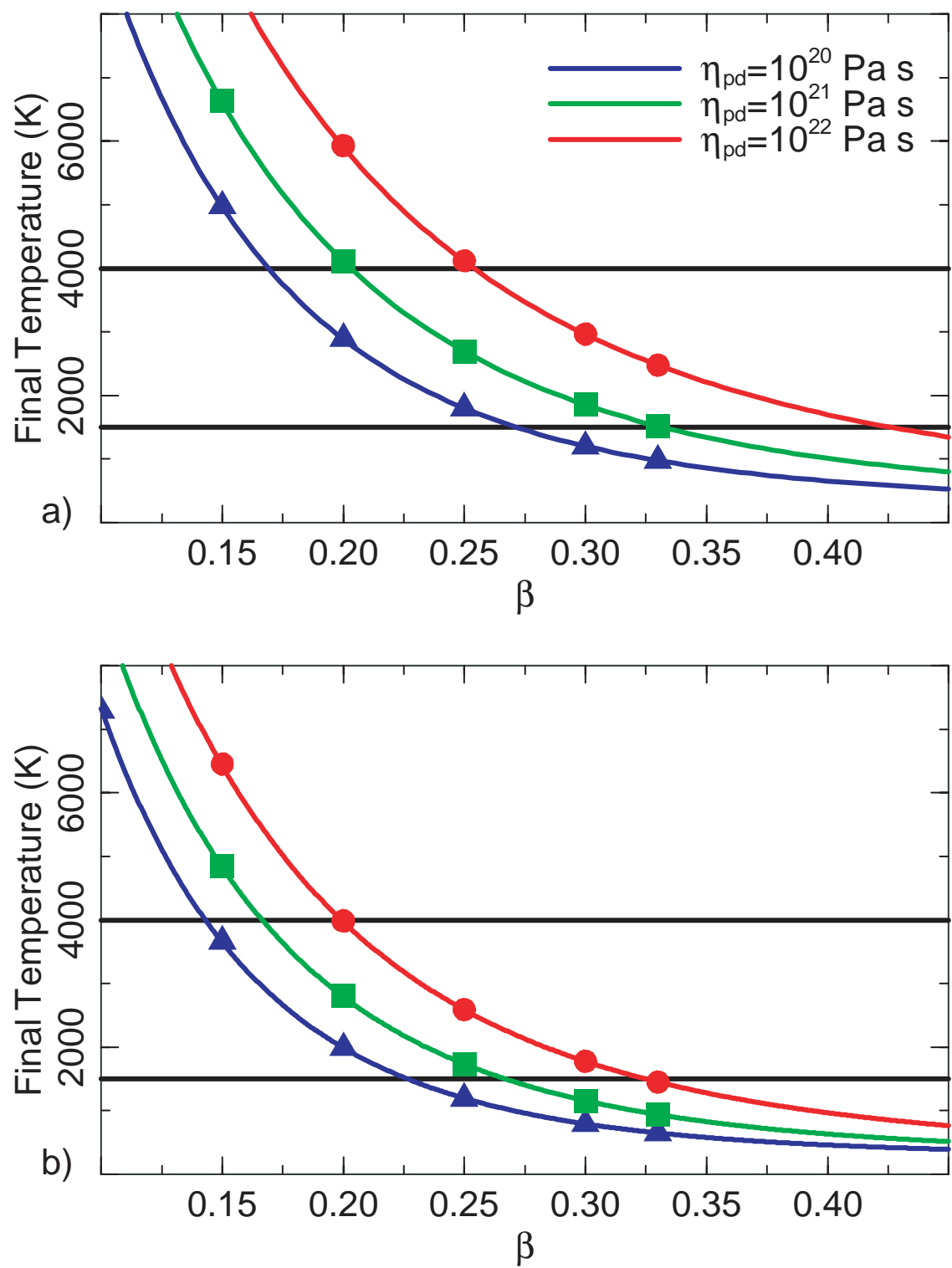

Figure 2. Whole-mantle convection results for two different values of critical Rayleigh number, (a) $R a_{c}=$ 1100 and (b) $R a_{c}=87.79$, in which final temperature is plotted against $\beta$. The analytical solutions for the three constrained values of present-day viscosity, $\eta_{p d}$ are plotted as curves. Symbols represent final temperatures obtained from the parameterized convection calculations. Triangles, squares, and circles represent results for $\eta_{p d}=10^{20}, 10^{21}$, and $10^{22} \mathrm{~Pa} \mathrm{~s}$, respectively.

[28] The dominant conclusion of this part of our study is that there is a wide range of parameters that allows the temperature of the mantle to remain within acceptable temperature limits. In the following section, it is shown that this is quite different for the case of layered convection in which only a narrow range of parameters produces acceptable layer temperatures. 
Table 2. Results Obtained From Whole-Mantle Parameterized Convection Calculations That Satisfy Our Temperature Constraints

\begin{tabular}{|c|c|c|c|c|c|c|c|c|c|}
\hline$R a_{c}$ & $E, \mathrm{~kJ} / \mathrm{mol}$ & $\eta_{p d}, \mathrm{~Pa} \mathrm{~s}$ & $\beta$ & {$[\mathrm{U}], \mathrm{pbb}$} & BSE, \% & Urey Ratio & $T_{\text {final }}, \mathrm{K}$ & $T_{\max }, \mathrm{K}$ & $\eta_{o}, \mathrm{~Pa} \mathrm{~s}$ \\
\hline 1100 & 0 & $10^{20}$ & 0.20 & 16.4 & 78.3 & 0.41 & 2892 & 3287 & $10^{20}$ \\
\hline 1100 & 0 & $10^{20}$ & 0.25 & 6.0 & 28.6 & 0.15 & 1800 & 3201 & $10^{20}$ \\
\hline 1100 & 0 & $10^{21}$ & 0.25 & 14.1 & 67.1 & 0.35 & 2694 & 3225 & $10^{21}$ \\
\hline 1100 & 0 & $10^{21}$ & 0.30 & 6.8 & 32.4 & 0.17 & 1856 & 3201 & $10^{21}$ \\
\hline 1100 & 0 & $10^{21}$ & 0.33 & 6.8 & 32.4 & 0.17 & 1519 & 3201 & $10^{21}$ \\
\hline 1100 & 0 & $10^{22}$ & 0.30 & 17.5 & 83.2 & 0.43 & 2962 & 3316 & $10^{22}$ \\
\hline 1100 & 0 & $10^{22}$ & 0.33 & 12.0 & 57.0 & 0.30 & 2479 & 3201 & $10^{22}$ \\
\hline 1100 & 525 & $10^{20}$ & 0.20 & 25.1 & 119.4 & 0.62 & 2882 & 3292 & $3.1 \times 10^{11}$ \\
\hline 1100 & 525 & $10^{20}$ & 0.25 & 35.2 & 167.5 & 0.87 & 1801 & 3201 & $5.9 \times 10^{4}$ \\
\hline 1100 & 525 & $10^{21}$ & 0.25 & 28.5 & 135.8 & 0.71 & 2691 & 3203 & $6.4 \times 10^{10}$ \\
\hline 1100 & 525 & $10^{21}$ & 0.30 & 35.7 & 170.1 & 0.88 & 1858 & 3201 & $1.7 \times 10^{6}$ \\
\hline 1100 & 525 & $10^{21}$ & 0.33 & 37.4 & 178.2 & 0.93 & 1514 & 3201 & $7.7 \times 10^{2}$ \\
\hline 1100 & 525 & $10^{22}$ & 0.30 & 28.6 & 136.0 & 0.71 & 2955 & 3313 & $5.3 \times 10^{12}$ \\
\hline 1100 & 525 & $10^{22}$ & 0.33 & 32.8 & 156.4 & 0.81 & 2481 & 3201 & $8.8 \times 10^{10}$ \\
\hline 87.79 & 0 & $10^{20}$ & 0.15 & 27.2 & 129.5 & 0.67 & 3662 & 3755 & $10^{20}$ \\
\hline 87.79 & 0 & $10^{20}$ & 0.20 & 6.1 & 32.4 & 0.17 & 1993 & 3201 & $10^{20}$ \\
\hline 87.79 & 0 & $10^{21}$ & 0.20 & 15.1 & 72.0 & 0.37 & 2790 & 3250 & $10^{21}$ \\
\hline 87.79 & 0 & $10^{21}$ & 0.25 & 5.9 & 28.0 & 0.14 & 1737 & 3201 & $10^{21}$ \\
\hline 87.79 & 0 & $10^{22}$ & 0.20 & 31.9 & 151.8 & 0.78 & 3981 & 4018 & $10^{22}$ \\
\hline 87.79 & 0 & $10^{22}$ & 0.25 & 12.9 & 61.4 & 0.32 & 2590 & 3207 & $10^{22}$ \\
\hline 87.79 & 0 & $10^{22}$ & 0.30 & 6.5 & 30.7 & 0.16 & 1775 & 3201 & $10^{22}$ \\
\hline 87.79 & 525 & $10^{20}$ & 0.15 & 27.5 & 130.9 & 0.68 & 3659 & 3773 & $3.2 \times 10^{12}$ \\
\hline 87.79 & 525 & $10^{20}$ & 0.20 & 32.1 & 153.0 & 0.80 & 1984 & 3201 & $1.5 \times 10^{6}$ \\
\hline 87.79 & 525 & $10^{21}$ & 0.20 & 25.7 & 122.4 & 0.63 & 2799 & 3256 & $1.6 \times 10^{11}$ \\
\hline 87.79 & 525 & $10^{21}$ & 0.25 & 35.5 & 168.9 & 0.88 & 1734 & 3201 & $1.5 \times 10^{5}$ \\
\hline 87.79 & 525 & $10^{22}$ & 0.20 & 30.8 & 146.9 & 0.76 & 3971 & 4027 & $1.2 \times 10^{15}$ \\
\hline 87.79 & 525 & $10^{22}$ & 0.25 & 29.3 & 114.0 & 0.72 & 2583 & 3201 & $2.4 \times 10^{11}$ \\
\hline 87.79 & 525 & $10^{22}$ & 0.30 & 36.0 & 171.3 & 0.89 & 1774 & 3201 & $3.5 \times 10^{6}$ \\
\hline
\end{tabular}

$R a_{c}$ is the critical Rayleigh number, $E$ is the activation energy, $\eta_{p d}$ is the constrained present-day viscosity, $\beta$ is the power law exponent, $[\mathrm{U}]$ is the uranium concentration required to satisfy present-day heat loss, BSE is the required [U] with respect to the bulk silicate Earth (21 ppb, [Van Schmus, 1995]), the Urey ratio is the present-day ratio of heat production to heat loss, $T_{\text {final }}$ is the final temperature, $T_{\max }$ is the maximum temperature, and $\eta_{\mathrm{o}}$ is the viscosity prefactor required to satisfy present-day viscosity constraints.

\section{Layered Mantle Convection}

\subsection{Model Setup}

[29] In layered convection models we need careful treatment of the inner boundary layer that will develop, with particular attention to the lower thermal boundary layer in the top layer. The temperature dependence of viscosity produces an asymmetry in the thicknesses of the upper and lower thermal boundary layers of a convecting layer, causing the lower thermal boundary layer to be thinner than the upper 
one. In addition, the presence of internal heating further reduces the thickness of the lower thermal boundary layer. We follow the method of Spohn and Schubert [1982] by treating the layered model in two distinct ways that differ only in the treatment of the lower thermal boundary layer of the top layer. For the layered model we perform one set of calculations that includes both upper and lower thermal boundary layers that are symmetrical in thickness. The other set of calculations is made with a vanishing lower thermal boundary layer in the top convecting layer. The two situations are sketched in Figure 3. We stress that neither of these thermal boundary layer configurations are directly applicable to the real Earth, which should yield results somewhere between these two end-member cases. The case in which the thermal boundary layers of symmetrical thickness are placed in the top convecting layer mimics isoviscous convection heated entirely from below, resulting in an upper bound on the insulating nature of the boundary between the two convecting layers. Alternatively, the case in which the lower thermal boundary layer of the top convecting layer is removed mimics convection heated entirely from within, placing a lower bound on the insulating nature of the interlayer boundary. We expect that results relevant to a layered Earth model fall between these two end-member cases because the top convecting layer would include components of heating both from within and below, so analysis of the two end-member cases allows us to adequately constrain values associated with the Earth.

[30] There are a number of ways to treat the core in parameterized convection models. One possibility is to calculate the heat flux from the core by calculating the heat flow across the lower thermal boundary layer [e.g., Spohn and Schubert, 1982]. This method allows a separate thermal history of the core but assumes that the thermal boundary layers
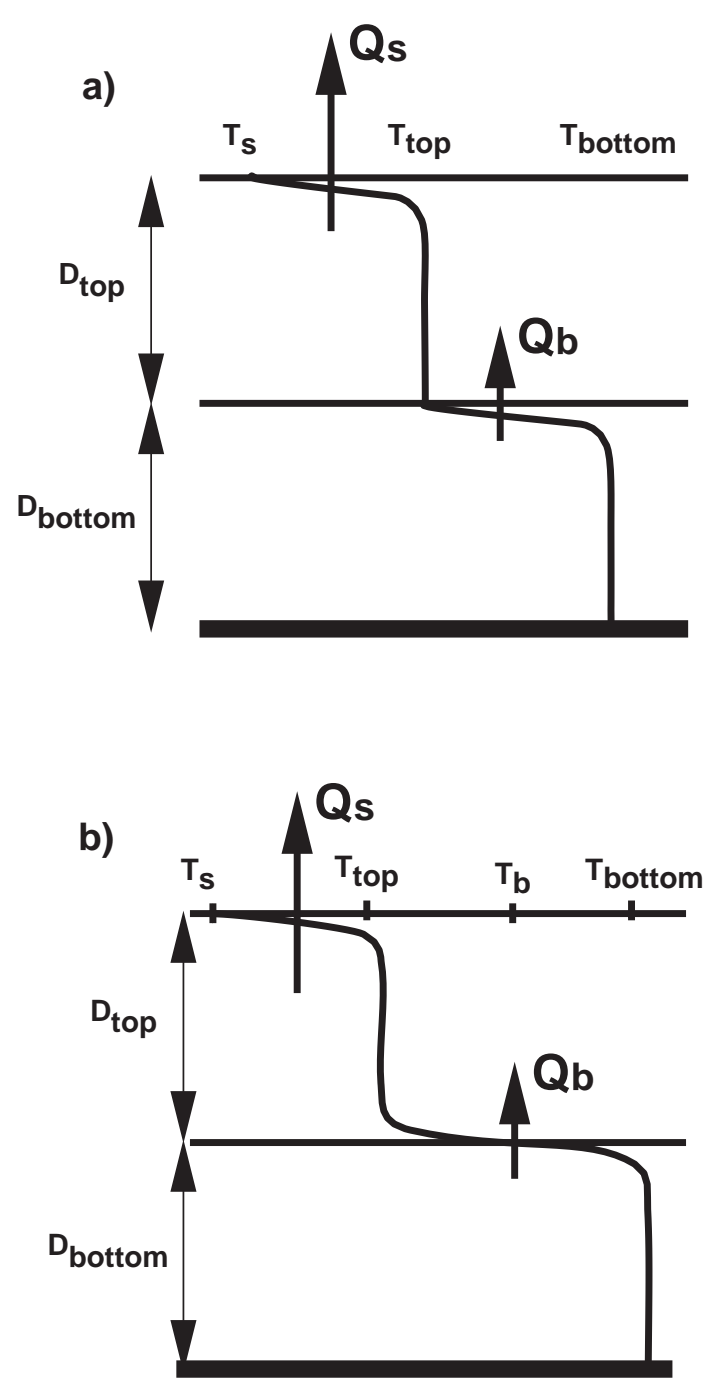

Figure 3. Schematic sketches of the thermal profiles under the two treatments of the lower thermal boundary layer of the top layer: (a) this thermal boundary layer is removed, and (b) this thermal boundary layer is equal in thickness to the upper boundary layer of the top layer. $Q_{s}$ and $Q_{b}$ are the heat flows at the surface and boundary between the layers, respectively. $D_{\text {top }}$ and $D_{\text {bottom }}$ are the thicknesses of the top and bottom layers, respectively. $T_{s}, T_{\text {top }}, T_{b}$, and $T_{\text {bottom }}$ are the temperatures of the surface, top layer, boundary between the layers, and bottom layer, respectively.

at the top and bottom of the lower mantle layer are of equal thickness unless the lower 
thermal boundary layer thickness is reduced by utilizing a local stability criterion as used by Stevenson et al. [1983]. Another possibility is to couple the core and mantle, maintaining them at thermal equilibrium [e.g., Honda, 1995; Honda and Iwase, 1996]. We choose to use a third possibility that imposes an insulated boundary condition at the base of the mantle [e.g., Stacey, 1980; Jackson and Pollack, 1984; Williams and Pan, 1992], which is a reasonable approximation because the heat flow from the core into the mantle is assumed to be small with respect to the heat production of the mantle [Davies, 1998].

[31] In the treatment of a vanishing lower thermal boundary layer in the top layer, the energy balance equation (4) is solved for both layers. In the bottom layer, $q_{i n}$ is set equal to zero owing to the insulated bottom boundary condition, and for the top layer, $q_{\text {in }}$ is set equal to $q_{\text {out }}$ calculated for the bottom layer.

[32] The equations are more complicated for the treatment in which the top layer includes a thermal boundary layer at its base. In this case the temperature between the layers, $T_{b}$, must be calculated. The heat flow across each boundary layer is calculated as follows:

$$
\begin{gathered}
q_{\text {top }, \text { out }}=\frac{K\left(T_{\text {top }}-T_{s}\right)}{D_{\text {top }}}\left(\frac{R a_{\text {top }}}{R a_{c}}\right)^{\beta}, \\
q_{\text {top }, \text { in }}=\frac{K\left(T_{b}-T_{\text {top }}\right)}{D_{\text {top }}}\left(\frac{R a_{\text {top }}}{R a_{c}}\right)^{\beta}, \\
q_{\text {bot,out }}=\frac{K\left(T_{\text {bot }}-T_{b}\right)}{D_{\text {bot }}}\left(\frac{R a_{\text {bot }}}{R a_{c}}\right)^{\beta}, \\
q_{\text {bot,in }}=0,
\end{gathered}
$$

where the Rayleigh numbers for each layer are

$$
R a_{\text {top }}=\frac{\alpha_{\text {top }} g \rho_{\text {top }}\left(T_{b}-T_{s}\right) D_{\text {top }}^{3}}{\kappa_{\text {top }} \eta\left(T_{\text {top }}\right)},
$$

$$
R a_{\mathrm{bot}}=\frac{\alpha_{\mathrm{bot}} g \rho_{\mathrm{bot}}\left(T_{\mathrm{bot}}-T_{b}\right) D_{\mathrm{bot}}^{3}}{\kappa_{\mathrm{bot}} \eta\left(T_{\mathrm{bot}}\right)} .
$$

[33] The value of $T_{b}$ may be determined by the necessary condition:

$$
q_{\mathrm{bot}, \mathrm{out}}=q_{\mathrm{top}, \mathrm{in}} \text {. }
$$

[34] The presence of a lower thermal boundary layer in the top layer is expected to restrict the overall transport of heat from the bottom layer to the top layer owing to the increase in total boundary layer thickness between the convecting layers.

[35] We start with radioactive element ratios provided by Kellogg et al. [1999] for each layer (see Table 1). Kellogg et al. [1999] use the present-day uranium concentrations of 7 and $25.6 \mathrm{ppb}$ in the top and bottom layers, respectively. We note that these values lead to an integrated present-day heat production of less than $15 \mathrm{TW}$, which would require a large degree of secular cooling. As a consequence, we modify the uranium concentrations of the bottom layer while keeping $\mathrm{K} / \mathrm{U}$ and $\mathrm{Th} / \mathrm{U}$ constant. We will show that for a temperature-dependent rheology and depleted top layer we must increase the uranium concentration significantly over $25.6 \mathrm{ppb}$ in order to achieve the the present-day heat loss. To maintain the depleted nature of the top layer, we use their exact concentration for the top layer of our models. Following from Kellogg et al. [1999], we also impose a $5 \%$ density contrast between both layers, which plays only a minor role in the determination of the heat capacity and Rayleigh number.

\subsection{Layered Results}

[36] Figure 4 shows the thermal evolution of two parameterized convection runs utilizing the layered geometry. These differ only in their treatment of the lower thermal boundary layer in the top layer. For the case with the vanishing thermal boundary layer in the top 

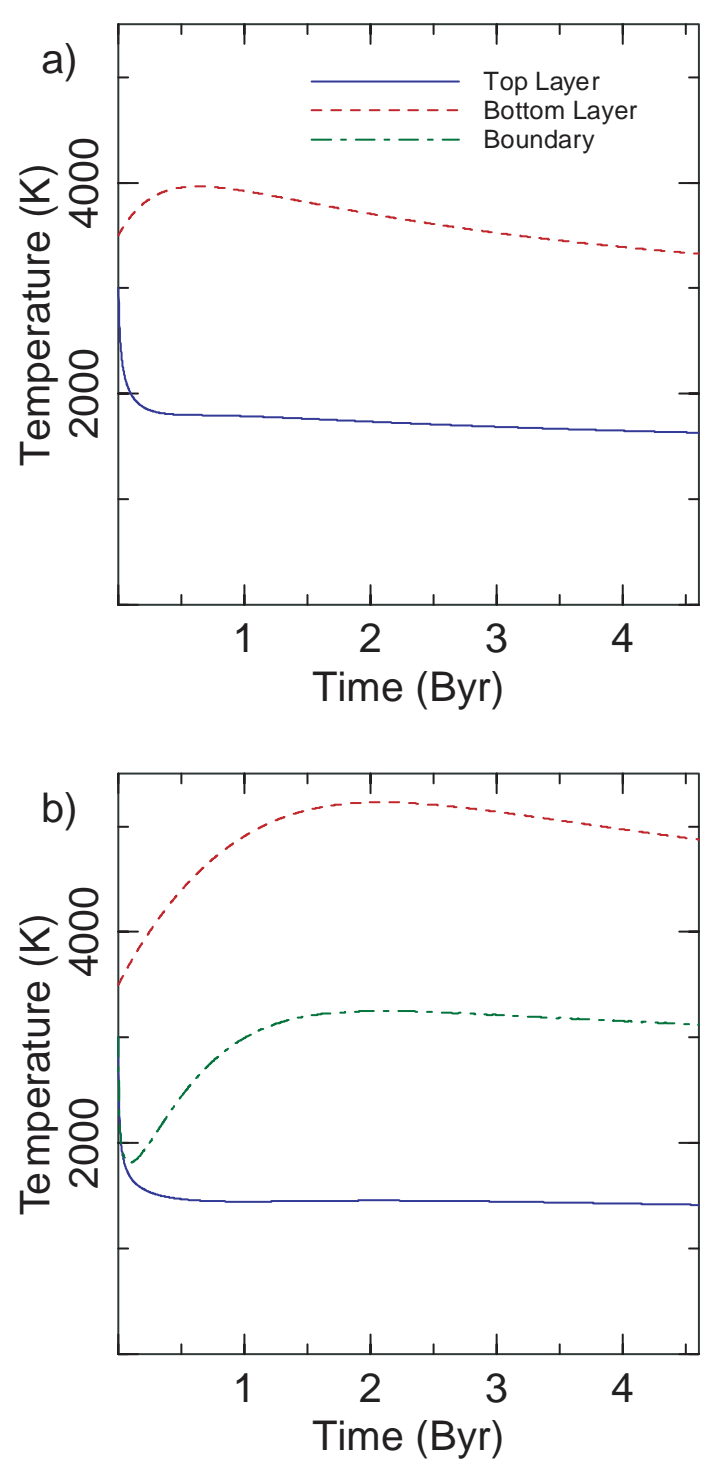

Figure 4. Temperature results obtained from layered parameterized convection calculations in which the boundary between the layers is placed at $1600-\mathrm{km}$ depth for two treatments of the lower thermal boundary layer of the top layer. For both, $R a_{c}=1100, \eta\left(\mathrm{T}_{p d}\right)=10^{20} \mathrm{~Pa} \mathrm{~s}$, and $\beta=0.25$. (a) Top and bottom layer temperatures are plotted for the treatment in which this thermal boundary layer is removed. (b) Temperatures for the top and bottom layers as well as the temperature of the boundary between the layers are plotted for the treatment in which symmetrical thermal boundary layers are included in the top layer. layer, the top layer experiences a brief period of rapid cooling followed by a prolonged stage of slow cooling up to the present day. The bottom layer of the same run undergoes some initial heating followed by cooling; yet these temperature results satisfy our temperature constraints. In contrast, the run that includes both boundary layers in the top layer experiences a more rapid initial cooling of the top layer and more intense heating of the bottom layer, both effects due to the greater thickness of the thermal boundary layer. As a result, temperature constraints are not satisfied.

[37] Comprehensive layering results are listed in Figure 5 for both treatments of the lower thermal boundary layer of the top layer. Results are separated into three categories based on whether a layer gets too hot (indicated by red circles), whether the top layer is too cool (blue squares), or whether the temperature constraints are satisfied (green asterisks). The bottom layer is judged as too hot if at any time during its history the temperature exceeded $4000 \mathrm{~K}$, and the top layer is considered too cool only if its present-day temperature is less than $1500 \mathrm{~K}$.

[38] It is observed that for a majority of cases the insulating effect of the thermal boundary layer imposed between the two convecting layers results in excessive bottom layer temperatures. The results also indicate that although reduced bottom layer temperatures may be produced by an increase in cooling efficiency provided by higher values of $\beta$ and/or lower present-day mantle viscosities, it often results in top layer temperatures that are too cool.

[39] In fact, very few cases provide temperatures that fall within the acceptable range, and those that do are only observed for cases in which the lower thermal boundary layer of the top layer has been removed. When symmetrical 


\section{Vanishing Lower}

\section{Symmetric TBLs in Top Layer}
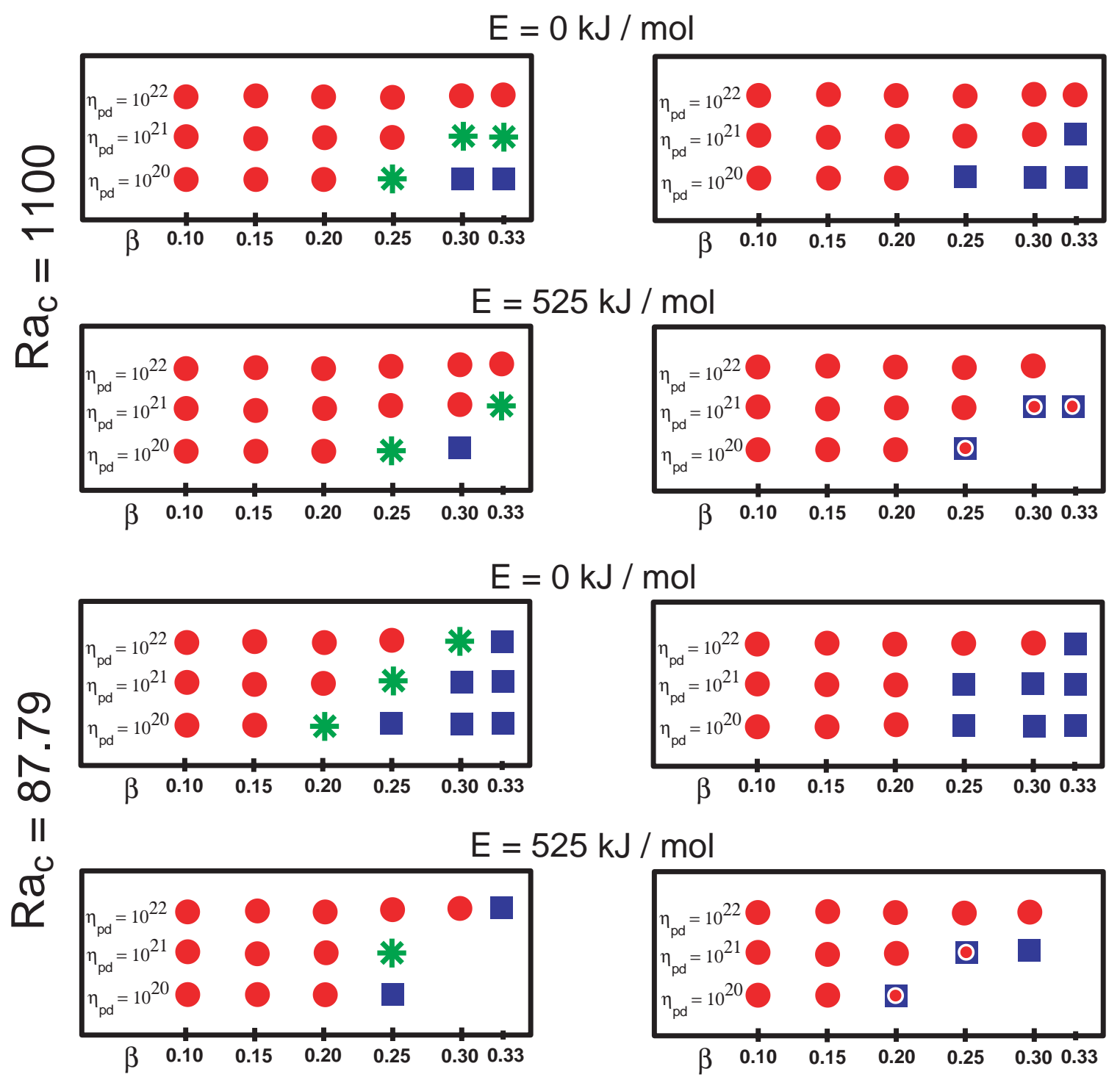

Figure 5. Parameterized convection results for the layered system in which the boundary between the layers is placed at a depth of $1600 \mathrm{~km}$. Red circles represent cases in which the bottom layer has exceeded $4000 \mathrm{~K}$ at some point during its thermal history. Blue squares represent cases in which the top layer has a final temperature below $1500 \mathrm{~K}$. Green asterisks represent cases which satisfy our temperature constraints.

boundary layers are are included within the top layer, the heat loss from the lower layer is significantly reduced. This results in cases in which the lower layer is too hot and the upper layer is too cool simultaneously (these are shown as circles within squares). 
Table 3. Results Obtained From Layered Parameterized Convection Calculations That Satisfy Our Temperature Constraints

\begin{tabular}{cccccccccccc}
\hline$R a_{c}$ & $\begin{array}{c}E, \mathrm{~kJ} / \\
\mathrm{mol}\end{array}$ & $\begin{array}{c}\eta_{p d}, \\
\mathrm{~Pa} \mathrm{~s}\end{array}$ & $\beta$ & $\begin{array}{c}{[\mathrm{U}],} \\
\mathrm{pbb}\end{array}$ & $\begin{array}{c}\text { BSE, } \\
\%\end{array}$ & $\begin{array}{c}\text { Urey } \\
\text { Ratio }\end{array}$ & $\begin{array}{c}T_{\text {final }}^{\text {top }}, \\
\mathrm{K}\end{array}$ & $\begin{array}{c}T_{\text {final }}^{\text {bottom }} \\
\mathrm{K}\end{array}$ & $\begin{array}{c}T_{\text {max }}^{\text {bottom }} \\
\mathrm{K}\end{array}$ & $\begin{array}{c}\eta_{\mathrm{o}}^{\text {top }}, \\
\text { Pa s }\end{array}$ & $\begin{array}{c}\eta_{\mathrm{o}}^{\text {bottom }} \\
\text { Pa s }\end{array}$ \\
\hline 1100 & 0 & $10^{20}$ & 0.25 & 14.1 & 66.9 & 0.21 & 1642 & 2675 & 3581 & $10^{20}$ & $10^{20}$ \\
1100 & 0 & $10^{21}$ & 0.30 & 21.2 & 100.9 & 0.26 & 1792 & 3062 & 3749 & $10^{21}$ & $10^{21}$ \\
1100 & 0 & $10^{21}$ & 0.33 & 14.8 & 70.8 & 0.21 & 1516 & 2542 & 3580 & $10^{21}$ & $10^{21}$ \\
87.79 & 0 & $10^{20}$ & 0.20 & 13.6 & 65.0 & 0.21 & 1701 & 2716 & 3577 & $10^{20}$ & $10^{20}$ \\
87.79 & 0 & $10^{21}$ & 0.25 & 12.6 & 60.0 & 0.20 & 1582 & 2560 & 3553 & $10^{21}$ & $10^{21}$ \\
87.79 & 0 & $10^{22}$ & 0.30 & 18.6 & 88.4 & 0.24 & 1716 & 2899 & 3677 & $10^{22}$ & $10^{22}$ \\
1100 & 525 & $10^{20}$ & 0.25 & 89.3 & 424.8 & 0.79 & 1631 & 3326 & 3964 & $1.5 \times 10^{3}$ & $5.7 \times 10^{11}$ \\
1100 & 525 & $10^{21}$ & 0.33 & 97.8 & 465.6 & 0.86 & 1504 & 3134 & 3678 & $5.8 \times 10^{2}$ & $1.8 \times 10^{12}$ \\
87.79 & 525 & $10^{21}$ & 0.25 & 90.9 & 432.6 & 0.81 & 1568 & 3194 & 3837 & $3.2 \times 10^{3}$ & $2.6 \times 10^{12}$ \\
\hline
\end{tabular}

All of these cases are derived from the boundary layer treatment in which the lower thermal boundary layer of the top convecting layer is removed. $R a_{c}$ is the critical Rayleigh number, $E$ is the activation energy, $\eta_{p d}$ is the constrained present-day viscosity, $\beta$ is the power law exponent, $[\mathrm{U}]$ is the uranium concentration in the bottom layer required to satisfy present-day heat loss, percent BSE is the required [U] with respect to the bulk silicate Earth (21 ppb [Van Schmus, 1995]), the Urey ratio is the present-day ratio of heat production to heat loss, $T_{\text {final }}^{\text {top }}$ and $T_{\text {final }}^{\text {bottom }}$ are the final temperatures of the top and bottom layers, respectively, $T_{\max }^{\text {bottom }}$ is the maximum temperature of the bottom layer, and $\eta_{\mathrm{o}}^{\text {top }}$ and $\eta_{\mathrm{o}}^{\text {bottom }}$ are the viscosity prefactors of the top and bottom layers, respectively, required to satisfy present-day viscosity constraints.

[40] Similar to the whole-mantle convection results, a decrease in present-day mantle viscosity or a reduction in $R a_{c}$ tends to shift the results toward lower values of $\beta$. As before, an increase in activation energy has minimal influence on the final results. However, it does play an important role in the uranium concentrations required to produce the present-day heat loss and the resulting Urey ratio (Table 3 ).

[41] In addition, Table 3 reveals the large temperature contrasts across the intermantle thermal boundary layer, exceeding $1000 \mathrm{~K}$ in all but one case. This has important consequences for the temperature-dependent viscosity formulation within each layer. In order to maintain a uniform mantle viscosity in the temperaturedependent trials the viscosity prefactor of the bottom layer must be significantly greater than that of the top layer, exceeding 8 orders of magnitude. Constraining viscosity to increase in the lower mantle in line with observations [Mitrovica and Forte, 1997] would lead to even greater prefactor contrasts.
[42] It is clear that the parameter range that allows acceptable temperatures is extremely narrow compared to that of whole-mantle convection.

\section{Discussion}

[43] As illustrated in the results, whole-mantle convection satisfies our constraints for a fairly large parameter range. This admissible parameter range is significantly reduced for layered convection. Moreover, acceptable results are only observed in the boundary layer treatment in which we remove the lower thermal boundary layer of the top layer. Not only does the alternative treatment of including symmetrical boundary layers in the top layer fail to produce results that satisfy the constraints, but it often yields large temperature contrast across the intermantle boundary layer, sometimes producing bottom layers that are too hot and top layers that are too cool.

[44] Both treatments of the lower thermal boundary layer of the top layer are end-member 
models. If the Earth is layered, values are expected to fall between these two results. One may argue that Earth values should fall closer to the treatment in which symmetrical boundary layers are included because of the high degree of bottom heating expected for a depleted layer overlying an enriched one. However, the reducing effect of temperature-dependent rheology on lower thermal boundary layer thickness makes this inference less concrete. Nonetheless, it is expected that the treatment in which the lower thermal boundary layer of the top layer is removed produces lower bounds on the bottom layer temperature. Examination of the maximum bottom layer temperatures of the acceptable cases (Table 3 ) reveals high values, all ranging from 3500 to $4000 \mathrm{~K}$. Considering these as lower bounds leads us to speculate that any additional insulation provided by the lower thermal boundary layer of the top layer would increase these temperatures above the acceptable range.

[45] In our models we have maintained the same value of $\beta$ in both layers in order to limit the parameter space. Some qualitative inferences may be made regarding the possibility of a different value of $\beta$ for each convecting layer. It is expected that an increase in $\beta$ will reduce the insulating effect of the boundary layers, whereas a decrease in $\beta$ will promote more insulation. A scenario in which the top convecting layer has a lower $\beta$ than that of the bottom convecting layer is expected to significantly increase the parameter range which produces acceptable results. The temperature contrast between the layers would be reduced, reducing the number of cases in which the top layer is too cool and the bottom layer is too hot. On the other hand, the contrasting scenario (higher $\beta$ in top and lower $\beta$ in bottom) is expected to reduce the parameter range that produces acceptable temperatures. Given a choice between these scenarios, the latter is likely to prevail based on the work of Gurnis
[1989] and Christensen [1984, 1985a, 1985b]. Provided that a strong temperature-dependent rheology produces a thick, insulating lid on the top surface of a convecting layer, a lower value of $\beta$ is likely as shown by Christensen [1984, 1985a, 1985b]. As shown by Gurnis [1989], if this lid is broken by initiating subduction (similar to the Earth's lithosphere), the value of $\beta$ would be significantly increased.

[46] For a highly temperature-dependent rheology in a layered Earth we surmise that the bottom convecting layer may possess a thick insulating lid, necessitating a lower value of $\beta$ than the top convecting layer that contains a broken, subducting lid. If this is the case, models involving higher values of $\beta$ in the top layer and lower values in the bottom layer would be expected to reduce even further the parameter range that produces acceptable results.

[47] Our study is similar to that of Spohn and Schubert [1982], who studied the feasibility of layered convection models in which the boundary between the layers is located at $670-\mathrm{km}$ depth, although our study involves a different method of varying the parameters leading to somewhat different results. By allowing the heat production in both layers to vary, Spohn and Schubert [1982] have shown that in order to satisfy temperature, heat flow, and viscosity constraints the bottom layer must be depleted in radioactive heat production with respect to the top layer. Aside from testing a deeper boundary at $1600-\mathrm{km}$ depth, our study differs in that we keep the amount of heat production in the top layer fixed, necessitating a higher degree of heat production in the bottom layer in order to satisfy the heat flow. We have allowed both viscosity prefactors and the bottom layer heat production to vary in each calculation in order to satisfy constraints. Our results differ from Spohn and Schubert [1982] in that we have generated a few model results that are consid- 
ered acceptable as well as many results that are considered too cool while maintaining an enriched bottom layer and a depleted top layer, in line with the model proposed by Kellogg et al. [1999]. Although our method and results differ somewhat, the conclusions are essentially the same; it is extremely difficult to reconcile layered mantle models with geophysical observations using a parameterized convection approach.

[48] It has been suggested that the resultant Urey ratios as well as required radioactive element concentrations may be used to further constrain our models. We have decided against this for several reasons. One reason involves the uncertainty in these values; we do not regard them to be as well constrained as our current constraints of present-day heat flow and mantle viscosity. Another reason is, as already mentioned, that both the Urey ratio and required uranium concentration depend heavily upon our choice of activation energy, although our final temperature results appear to be relatively decoupled from this choice. Because we do not claim to know the effective activation energy of the Earth (but assume it falls somewhere between our end-member values], we can only say that the resulting Urey ratios and uranium concentrations provide only end-member constraints and Earth-like values are likely found in between. In fact, the Urey ratios resulting from our calculations should be viewed as an estimate only because other studies have concluded that correcting for the contraction of the Earth upon cooling [Stacy, 1981; Spohn and Breuer, 1993] and growth of the continental crust [Breuer and Spohn, 1993; Spohn and Breuer, 1993] tends to reduce the resulting Urey ratios.

[49] While recognizing the limits of parameterized convection modeling, our results favor a mode of whole-mantle convection over layered convection in order to satisfy the constraints of heat flow, temperature, and viscosity.
Other models invoking distinct reservoirs [e.g., Becker et al., 1999] should be investigated more thoroughly as possibilities to explain the geochemistry of MORBs and OIBs.

\section{Conclusions}

[50] Layered convection models have been suggested to explain the geochemistry observed at the Earth's surface, but they are problematic in that layering tends to reduce the efficiency of mantle cooling. The parameterized convection study presented here was performed to examine the heat transport properties of the layered system proposed by Kellogg et al. [1999] and to investigate the parameter range in which this model is consistent with observed present-day heat loss, viscosity, and temperature constraints.

[51] Results show that whole-mantle models satisfy the above constraints under a wide range of parameters, whereas for most parameter combinations in the layered model the insulating nature of the thermal boundary layer induced between the two convecting layers acts to superheat the bottom layer to temperatures well above the melting temperature. Permissible bottom layer temperatures are achieved for high values of $\beta$ and low values of critical Rayleigh number and present-day viscosity, usually at the trade-off of producing top layer temperatures that are too cool. Only a narrow range of parameters produces results that fall within acceptable temperature limits. The layered results that do satisfy the constraints are only observed when the lower thermal boundary layer is removed, and because these are lower bound temperatures, it is expected that this narrow parameter range may be further reduced or disappear completely for a layered Earth. While recognizing that parameterized convection has its limitations, these results indicate that under the observational constraints of present-day heat loss and viscosity 
along with reasonable temperature constraints, whole-mantle convection provides the most likely mode of heat loss for the Earth.

\section{Acknowledgments}

[52] We thank Henry Pollack and Jamie Kellogg for helpful discussions and Tilman Spohn and two anonymous reviewers for constructive reviews.

\section{References}

Becker, T. W., J. B. Kellogg, and R. J. O'Connell, Thermal constraints on the survival of primitive blobs in the lower mantle, Earth Planet. Sci. Lett., 171, 351-365, 1999.

Bijwaard, H., and W. Spakman, Tomographic evidence for a narrow whole mantle plume below Iceland, Earth Planet. Sci. Lett., 166, 121-126, 1999.

Bijwaard, H., W. Spakman, and E. R. Engdahl, Closing the gap between regional and global travel time tomography, J. Geophys. Res., 103, 30,055-30,078, 1998.

Breuer, D., and T. Spohn, Cooling of the Earth, Urey ratios, and the problem of potassium in the core, Geophys. Res. Lett., 20, 1655-1658, 1993.

Christensen, U. R., Heat transport by variable viscosity convection and implications for the Earth's thermal evolution, Phys. Earth Planet. Inter., 35, 264-282, 1984.

Christensen, U. R., Heat transport by variable viscosity convection II: pressure influence, non-Newtonian rheology and decaying heat sources, Phys. Earth Planet. Inter., 37, 183-205, 1985a.

Christensen, U. R., Thermal evolution models of the Earth, J. Geophys. Res., 90, 2995-3007, 1985b.

Conrad, C. P., and B. H. Hager, The thermal evolution of an earth with strong subduction zones, Geophys. Res. Lett., 26, 3041-3044, 1999.

Davies, G. F., Topography: A robust constraint on mantle fluxes, Chem. Geol., 145, 479-489, 1998.

Davies, G. F., and M. A. Richards, Mantle convection, J. Geol., 100, 151-206, 1992.

Giannandrea, E., and U. Christensen, Variable viscosity convection experiments with a stress-free upper boundary and implications for the heat transport in the Earth's mantle, Phys. Earth Planet. Inter., 78, 139-152, 1993.

Grand, S. P., R. D. van der Hilst, and S. Widiyantoro, Global seismic tomography: A snapshot of convection in the Earth, GSA Today, 7, 1-7, 1997.

Gurnis, M., A reassessment of the heat transport by variable viscosity convection with plates and lids, Geophys. Res. Lett., 16, 179-182, 1989.
Hofmann, A. W., Mantle geochemistry: the message from oceanic volcanism, Nature, 385, 219-229, 1997.

Holland, K. G., and T. J. Ahrens, Melting of (Mg,Fe) $)_{2-}$ $\mathrm{SiO}_{4}$ at the core-mantle boundary of the Earth, Science, 275, 1623-1625, 1997.

Honda, S., A simple parameterized model of Earth's thermal history with the transition from layered to whole mantle convection, Earth Planet. Sci. Lett., 131, 357369, 1995.

Honda, S., and Y. Iwase, Comparison of the dynamic and parameterized models of mantle convection including core cooling, Earth Planet. Sci. Lett., 139, 133-145, 1996.

Jackson, M. J., and H. N. Pollack, On the sensitivity of parameterized convection to the rate of decay of internal heat sources, J. Geophys. Res., 89, 10,103-10,108, 1984.

Karato, S., and P. Wu, Rheology of the upper mantle: A synthesis, Science, 260, 771-778, 1993.

Kellogg, L. H., B. H. Hager, and R. D. van der Hilst, Compositional stratification in the deep mantle, Science, 283, 1881-1884, 1999.

McKenzie, D., and F. M. Richter, Parameterized thermal convection in a layered region and the thermal history of the Earth, J. Geophys. Res., 86, 11,667-11,680, 1981.

Mitrovica, J. X., and A. M. Forte, Radial profile of mantle viscosity: Results from the joint inversion of convection and post-glacial rebound observables, J. Geophys. Res., 102, 2751-2769, 1997.

Morgan, W. J., Convection plumes in the lower mantle, Nature, 230, 42-43, 1971.

Peltier, W. R., Mantle viscosity, in Mantle Convection: Plate Tectonics and Global Dynamics, edited by W. R. Peltier, pp. 389-478, Gordon and Breach Sci., New York, 1989.

Pollack, H. N., and D. S. Chapman, Mantle heat flow, Earth Planet. Sci. Lett., 34, 174-184, 1977.

Pollack, H. N., S. J. Hurter, and J. R. Johnson, Heat flow from the Earth's interior: Analysis of the global data set, Rev. Geophys., 31, 267-280, 1993.

Schubert, G., D. Stevenson, and P. Cassen, Whole planet cooling and the radiogenic heat source contents of the Earth and Moon, J. Geophys. Res., 85, 2531-2538, 1980.

Shen, Y., S. C. Solomon, I. T. Bjarnason, and C. J. Wolfe, Seismic evidence for a lower-mantle origin of the Iceland plume, Nature, 395, 62-65, 1998.

Spohn, T., and D. Breuer, Mantle differentiation through crust growth and renewal and the thermal evolution of the Earth, in Evolution of the Earth and Planets, Geophys. Monogr. Ser., vol. 74, edited by E. Takahashi, R. Jeanlozm, and D. Rubie, pp. 55-71, AGU, Washington, D. C., 1993. 
Spohn, T., and G. Schubert, Modes of mantle convection and the removal of heat from the Earth's interior, $J$. Geophys. Res., 87, 4682-4696, 1982.

Stacey, F. D., The cooling Earth: A reappraisal, Phys. Earth Planet. Inter., 22, 89-96, 1980.

Stacey, F. D., Cooling of the Earth - A constraint on paleotectonic hypotheses, in Evolution of the Earth, Geodyn. Ser., vol. 5, edited by R. J. O'Connell and W. S. Fyfe, pp. 272-276, AGU, Washington, D. C., 1981. Stevenson, D. J., T. Spohn, and G. Schubert, Magnetism and thermal evolution of the terrestrial planets, Icarus, 54, 466-489, 1983.

Tackley, P. J., Three-dimensional simulations of mantle convection with a thermo-chemical basal boundary layer: $\mathrm{D}^{\prime \prime}$, in The Core Mantle Boundary Region, Geodyn. Ser, vol. 28, edited by M. Gurnis, M. E. Wysession, E. Knittle, B. A. Buffett, pp. 231-253, AGU, Washington, D. C., 1998.

Turcotte, D. L., On the thermal evolution of the Earth, Earth Planet. Sci. Lett., 48, 53-58, 1980.

Turcotte, D., and G. Schubert, Geodynamics, 450 pp., John Wiley, New York, 1982. van der Hilst, R. D., and H. Karason, Compositional heterogeneity in the bottom 1000 kilometers of the Earth's mantle: Toward a hybrid convection model, Science, 283, 1885-1888, 1999.

van der Hilst, R. D., S. Widiyantoro, and E. R. Engdahl, Evidence for deep mantle circulation from global tomography, Nature, 386, 579-584, 1997.

van Keken, P. E., The art of maintaining a chemically diverse mantle,paper presented 12th annual IRIS workshop, Rockport, Maine, May 7-11, 2000.

Van Schmus, W. R., Natural Radioactivity of the crust and mantle, in Global Earth Physics, A Handbook of Physical Constants, AGU Ref. Shelf, vol. 1, edited by T. J. Ahrens, pp. 283-291, AGU, Washington, D.C., 1995.

Wang, Z. W., The melting of Al-bearing perovskite at the core-mantle boundary, Phys. Earth Planet. Inter., 115, 219-228, 1999.

Williams, D. R., and V. Pan, Internally heated mantle convection and the thermal and degassing history of the Earth, J. Geophys. Res., 97, 8937-8950, 1992.

Zerr, A., A. Diegeler, and R. Boehler, Solidus of the Earth's deep mantle, Science, 281, 243-246, 1998. 